\title{
Electronic Word-of-mouth Marketing in E-commerce based on Online Product Reviews
}

\author{
${ }^{1}$ PengPeng Zhang, ${ }^{2}$ Sheng Bin and ${ }^{1 *}$ Gengxin Sun

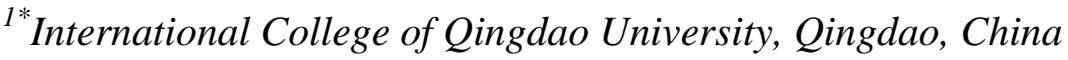 \\ ${ }^{2}$ Software Technical College of Qingdao University, Qingdao, China \\ ${ }^{1}$ rjxy@qdu.edu.cn, ${ }^{2}$ binsheng@qdu.edu.cn, ${ }^{1 *}$ sungengxin@qdu.edu.cn
}

\begin{abstract}
In E-commerce, purchase decisions of consumers are often strongly influenced by people who consumers know and trust, and word-of-mouth (WOM) is the most important factor when consumers are making a purchase decision. Many online consumers tend to wait for the opinions of early consumers before making a purchase decision to reduce the risk of buying products. So numerous E-commerce websites provide product reviews function for consumers to publicize their personal reviews of purchased products and thus facilitate word-of-mouth communication among consumers. This paper describes wordof-mouth, as a potentially cost-effective means for marketing, especially influence of opinion leaders in electronic word-of-mouth marketing.
\end{abstract}

Keywords: Word-of-mouth, E-commerce, opinion leaders, product reviews, online social network

\section{Introduction}

With the rapid development of E-commerce, more and more consumers choose shopping online. But browsing, searching, and buying a product on E-commerce websites becomes often a time consuming and frustrating operations for consumers. In reality, decision to buy a product is often strongly influenced by interpersonal influence, we far more likely to believe recommendations from people who we know and trust. In Ecommerce, we would like to take early consumer's reviews of purchased products into consideration. Online communities such as discussion forums and message boards, allow consumers to express their personal preferences and to share their personal evaluations of product performance by rating others' reviews. There are different kinds of online consumer review websites. First, online retailers such as Taobao.com and Amazon.com publish consumer reviews on their websites. Second, traditional consumer magazines sponsor forums. Third, independent consumer community intermediaries (e.g., douban.com) organize consumer reviews on various products.

These online consumer product reviews in above websites contain valid information for E-commerce companies. E-commerce companies have recently started to capture data on the interpersonal influence between consumers in their websites, with the potential objective of understanding and leveraging interpersonal influence in consumers' purchase decision making to improve customer relationship management and increase sales. Some E-commerce companies are attempting to support their potential consumers' decision making process by introducing personalized Web-based recommender systems. A typical collaborative filtering algorithm of recommender system builds consumer's neighborhood based on his preferences of shared products and weighs the interest of neighbors with similar taste to generate new recommendations [1]. But Sinha [2] found that consumers are far more likely to believe recommendations by electronic word-of-mouth. It means that friends and family-members rather than from automated recommender systems in Ecommerce websites are more credible. 
In online network, web users gradually began to know and trust each other through online social network. According to new research [3], online social network including Twitter and Facebook are driving an increasing volume of traffic to retail websites, and are thus becoming a starting point for Web users who are interested in E-commerce. The increasing traffic from online social network to online retailers shows that highly influential consumers directly affect other consumers' decision making. Therefore, Ecommerce companies can take advantage of this interpersonal influence between consumers to increase sales.

\section{Social Influence and Word-of-mouth}

Word-of-mouth communication is an important facilitator of vicarious learning and can have a large impact on consumer decisions [4-5]. Described as word-of-mouth (WOM) communication, the process allows consumers to share information and opinions that direct buyers towards and away from specific products and services [6].

Research on WOM can date to the 1960s [7-9], and WOM definitions have evolved over time [10]. In the early years, WOM was defined as face-to-face communication about products or companies between people [7, 10]. Later, WOM was described more broadly to include all informal communications directed at other consumers about the characteristics of particular goods and services or their sellers. Those informal communications clearly indicated that these are the communications of interpersonal relationships, as opposed to those through mass-media channels that pass product knowledge from producers or providers to consumers. Recently, WOM was argued that it can be mediated by electronic means. It was so-called electronic word-of-mouth [11]. The definitional evolution indicates that WOM is becoming both more pervasive with today information technology ubiquitous.

Before considering WOM for marketing, there are a few general questions that should be answered to add to our understanding:

(1) Why do consumers spread WOM? It indicated that positive and negative feelings associated with a product experience which needs call for a discharge in the form of WOM. So a consumer's affective elements of satisfaction, pleasure, and sadness would motivate consumers to wish to share experiences with others. More than 10000 rare coin auctions from taobao are studied and found that taobao users, both buyers and sellers, exhibited reciprocity toward those other users who had rated them previously, and that they were more likely to rate their trading partners in order to increase chances of their being reciprocally rated.

(2) Where does WOM originate? The key WOM originator is the opinion leader, an active user who interprets the meaning of media message content for others. Opinion leaders are interested in particular product fields, make an effort to expose their product reviews to mass media sources, and are trusted by opinion followers to provide knowledgeable advice [12-13].

(3) What are the outcomes from the dissemination of WOM? Unsurprisingly, positive WOM increases the probability of purchase, while negative WOM has the opposite effect. Some recent researches determined that electronic WOM impacted not only the receiver's perceived value of a company's products, but also their loyalty intentions [14]. Thus, the exchange of product information through WOM reduces producer-consumer information asymmetries, which ultimately results in an acceleration or deceleration of product acceptance.

The above questions can be concluded to procedures of WOM, as shown in Figure 1. 


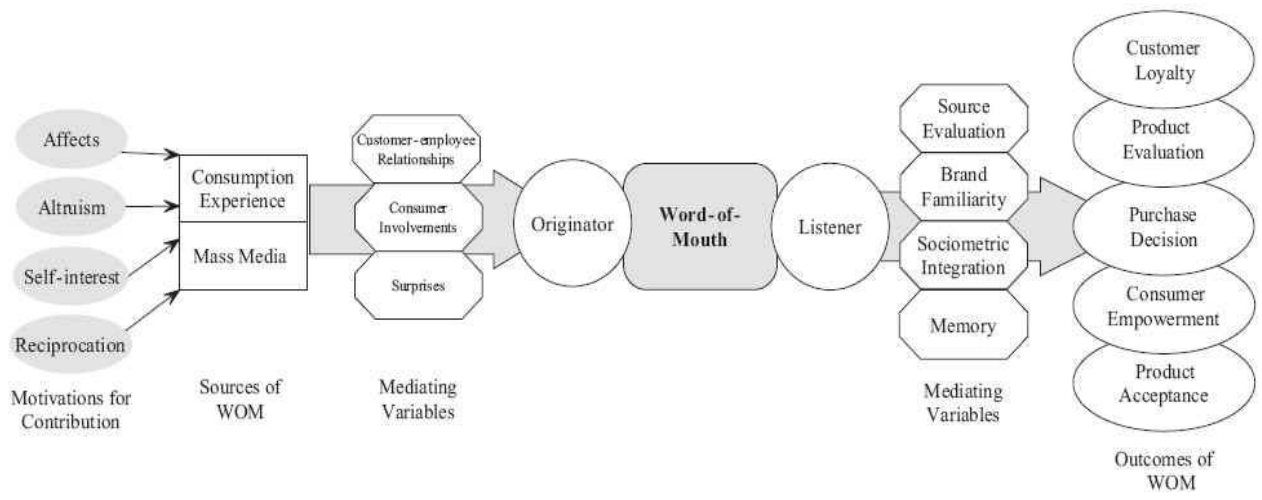

Figure 1. The Procedures of Word-of-mouth

Online social network provides various methods for participants who can interact, exchange opinions, and compare experiences with others. It can be defined as a graph of relationships and interactions within a group of individuals, which often plays a fundamental role as a medium for the spread of information and influence among others. There have been some researches focused on the consumer networks that are formed through the direct and indirect interactions between consumers to maximize the impact of direct marketing through social influence. Models have been proposed to identify a set of highly influential consumers to maximize word-of-mouth effects or to find target consumers based on the preferences and influential impact from previous consumers [1517].

The methods using existing consumers to identify potential consumers who are likely to buy, have started to incorporate social influence in E-commerce. But it has been limited to data sources about social interaction captured from E-commerce websites, because of only a subset of the information becoming available. In this paper, we analyze the impact of social influence in E-commerce decision making. We also survey current technology for online social network analysis which could be adopted and modified for analysis of Ecommerce social interactions. At last, we provide a specific algorithm for detecting opinion leaders in E-commerce websites, our understanding of social influence in Ecommerce could be a starting point to develop methodologies for a social interaction based E-commerce decision making system.

\section{E-Commerce Decision Making Process}

In this section we describe a consumer's decision making process in E-commerce and describe how social influence affects each decision making step. In general, consumers' decision making process consist of Need recognition, Information search, Evaluation, Purchase and After purchase evaluation [18]. For instance, consider a consumer who wants to buy a book in an online shopping website. Stage1: The consumer recognizes a need to buy a book. He has to decide if he will go to Amazon.com where he shops often, or try another online store such as Taobao.com. Sometimes, he also decides if it is worthwhile to visit a online network community site such as Epinions.com in advance to search for information about a product. Stage $2 \&$ 3: After deciding to visit Amazon.com, he begins to search for a book. He reads book sample pages, consumers' reviews about a few books and compares them based on his preference, consumers' reviews and reviews' ratings, as evaluated by other consumers. At this point, he has to determine which book to buy and decide whether he would like to rate some reviews that were helpful in making a purchase decision. Stage 4: After choosing which book to buy, he has to decide whether he should buy the book at Amazon.com or some other shopping websites. Stage 5: When he decides to place an order at Amazon.com, he would be recommended a set of books, which were frequently purchased together. He must decide whether to buy the 
recommended books or not. Stage 6: After reading the book which he bought, the consumer considers writing a review to share his opinion and to help other consumers' decision making. He would also decide to rate some reviews that influenced his purchase decision of the product based on the reviews' accuracy or honesty. Stage 7: He would also decide whether or not he should rate the book. The complete stages can be shown in Figure 2.

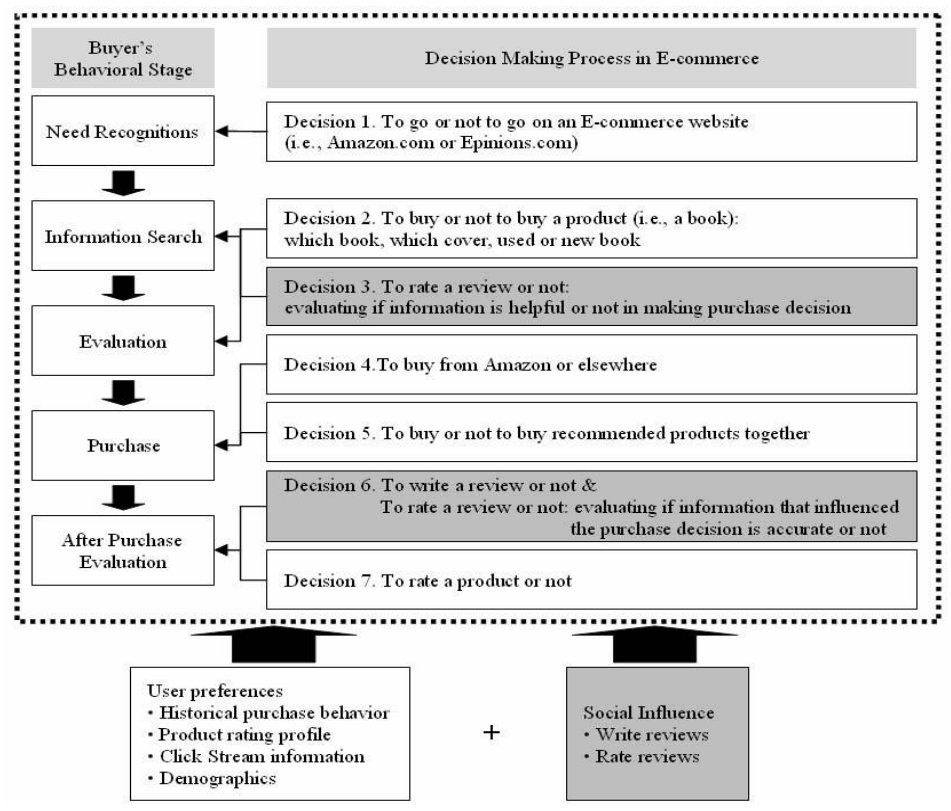

Figure 2. Decision Making Process in E-Commerce

In consumer' decision making process, social influence plays an important role. In Ecommerce these social interactions, which manifest that consumers write reviews and rate reviews written by other consumers, can consist of online social network, which would has an effect on every decision stage. On Need recognition stage, E-commerce websites could anticipate a consumer's latent purchase needs based on social community or group in an online social network to which a consumer belongs and the relationship with which a consumer associates. Then, E-commerce websites can stimulate latent purchase needs by sending recommendation information which encourages a consumer to visit its website. On Information search \& Evaluation stages, the consumer needs more help than at any other decision stage, because the consumer wants to reduce the risk of buying a new product and effectively make a satisfactory decision. Based on opinion leaders' ratings in a product category or friends or trusted members' ratings, the search space which has a small set of products to be compared could be reduced. Most Ecommerce websites also encourage consumers to write review and rate reviews written by others; therefore, they can identify influential consumers called opinion leaders based on how many reviews the consumers wrote and how much credit the consumers receive. On Purchase stage, some online social network websites such as Epinions.com an Douban.com also allow a consumer to evaluate E-commerce websites, E-commerce websites can offer a consumer the reviews of its website which previous consumers wrote to build trust in the Ecommerce website or the product provider. On After purchase evaluation stage, Ecommerce websites could encourage a consumer to write a review or rate a product by recommending a relevant community about the product and informing them about what has happened in the community. People tend to participate in discussions when they experience opposite opinions, even wrong or different opinions, and get feedback on their opinions from others. 


\section{Detecting Opinion Leader Nodes in Online Product Reviews Network}

In this section, we firstly discuss technology for online product reviews network analysis which could be used to leverage social influence in the E-commerce decision making process. Then a new opinion leader nodes detecting algorithm is proposed.

\subsection{Technology for Online Product Reviews Network Analysis}

Product reviews network can be as online social network, online social network analysis is for analyzing patterns of relationships and interactions between network nodes in order to discover the underlying social structure.

Opinion leader was used to characterize a set of users that are ahead to others in terms of developing new product needs. The concept of opinion leader in social networks aims to understand users whose opinions are highly influenced by those opinion leaders. In online social network, opinion leader nodes always key nodes, in order to identify key nodes in online social networks, various centrality measures and algorithms, such as Degree, Betweenness, Closeness, PageRank and HITS, have been used. The key nodes often play a role by issuing information, ideas and influence, or bridging different communities. The removal of key nodes could disrupt a network more than common nodes. In other words, the key nodes could be effectively used to maximize social influence within online social network. Theses centrality measures and algorithms for key nodes can be used to rank consumers based on the amount of influence on consumers' purchase decision making and identify opinion leaders who often write reviews earlier than other consumers.

Detecting a community or a social group in a large network has also been important for network analysis. In an E-commerce environment, mining cohesive subgroups where consumers have relatively strong connections and similar purchase behavior or common interests is a key issue for marketers in order to maximize the word-of-mouth effect in an individual subgroup.

\subsection{Opinion Leader Nodes Detecting Algorithm in Online Product Reviews Network}

Because opinion leaders reflect the opinions of the masses and have strong influence on other consumers, opinion leader detecting has attracted more and more attention and E-commerce websites have investigated how to identify opinion leaders from review networks. In the field of opinion leader detecting and identifying, most existing work is focused on identifying opinion leaders at a global level [19-21]. But opinion leaders always have their personal expertise and interest. Therefore, we propose a new opinion leaders detecting algorithm in domain level, which would be more authoritativeness and representativeness using consumers' reviews and linking information among consumers.

The structure of product review network is shown as Figure 3. Consumers publish reviews which belong to different domains.

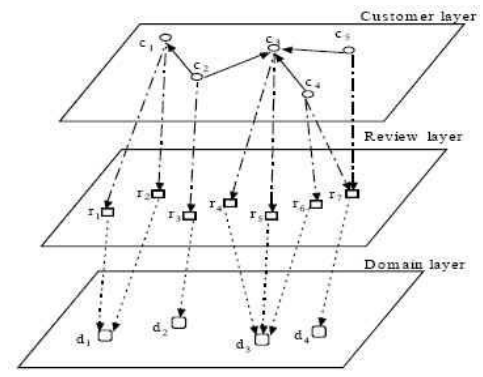

Figure 3. Layer Structure of Online Product Reviews Network 
For instance, customer $c_{1}$ published two reviews $r_{1}$ and $r_{2}$ in domain $d_{1}$, therefore domain $d_{1}$ is one element of $c_{1}$ 's expertise space.

In our algorithm, let $C=\left\{c_{1}, c_{2}, \ldots, c_{m}\right\}$ be the set of consumers, $D=\left\{d_{1}, d_{2}, \ldots d_{m}\right\}$ be the set of domains and $V_{m n}$ be the matrix which represents consumers' expertise. Each element $v_{i j}$ in $V_{m n}$ represents the number of reviews published by consumer $i$ which belong to domain $j$. Consumers' relationships are be used to construct a directed graph $G(V, E)$, where $V$ represents all the customers and $E$ refers to their relations.

Based on product reviews network acting as the directed graph, the algorithm based on random surfer model is described as follows: the random surfer visits each consumer with certain probability by following the appropriate edge in $G$, The random surfer performs domain-sensitive random walk. By doing so, we are essentially constructing a domainspecific graph among consumers. Given a domain $d$, each element in the transition matrix $P_{d}$, which represents the domain-specific transition probability of the random surfer from consumer $c_{i}$ to consumer $c_{j}$, is defined as follows:

$$
t(i, j)=T(i, j) * \operatorname{sim}_{d}(i, j)
$$

Where, if $i$ interests in $j, T(i, j)=1$, otherwise, $T(i, j)=0 . \operatorname{sim}_{d}(i, j)$ represents expertise similarity between consumer $c_{i}$ and $c_{j}$ in domain $d$. The more similar the two consumers' expertise is, the higher the transition probability from customer $c_{i}$ to $c_{j}$.

$$
\operatorname{sim}(i, j)=1-\left|V_{i d}^{\prime}-V_{j d}^{\prime}\right|
$$

Where, $V^{\prime}$ is a row of $V$. Eq. (2) means the difference of the two consumers' expertise in domain $d$.

We normalize $V$ according to row as $V^{\prime}$ and according to column as $V^{\prime \prime} . E_{d}$ is defined as the $d$-th column of matrix $V^{\prime \prime}$.

$$
E_{d}=V_{d}^{\prime \prime}
$$

Based on the domain-specific transition probability matrix $P_{d}$ and vector $E_{d}, C R_{d}$, the consumer rank in domain $d$, can be defined iteratively by Eq. (4).

$$
C R_{d}=\alpha^{*} P_{d} \times C R_{d}+(1-\alpha) * E_{d}
$$

Where $\alpha$ is the threshold value that can be used to adjust the contribution of $P_{d}$ and $E_{d}$.

\section{Experiment and Analysis}

In experiment, we collect experiment data from taobao.com. The dataset consists of three categories products shown in Table 1.

Table 1. Comparison of the Analysis Results of the Proposed Algorithm and PageRank Algorithm

\begin{tabular}{ccc}
\hline Domain & Product Number & Customer Number \\
\hline Clothes & 13509 & 3956 \\
Toy & 7018 & 1903 \\
Sports & 3938 & 1701 \\
\hline
\end{tabular}

To evaluate the effectiveness of our algorithm, two metrics, coverage and authority [21-22], are adopted, and two baseline algorithms, PageRank and Indegree-based 
algorithms have been compared with our algorithm. The authority and coverage change of different domains with the number of identified opinion leaders by different algorithms are shown in Figure 4.
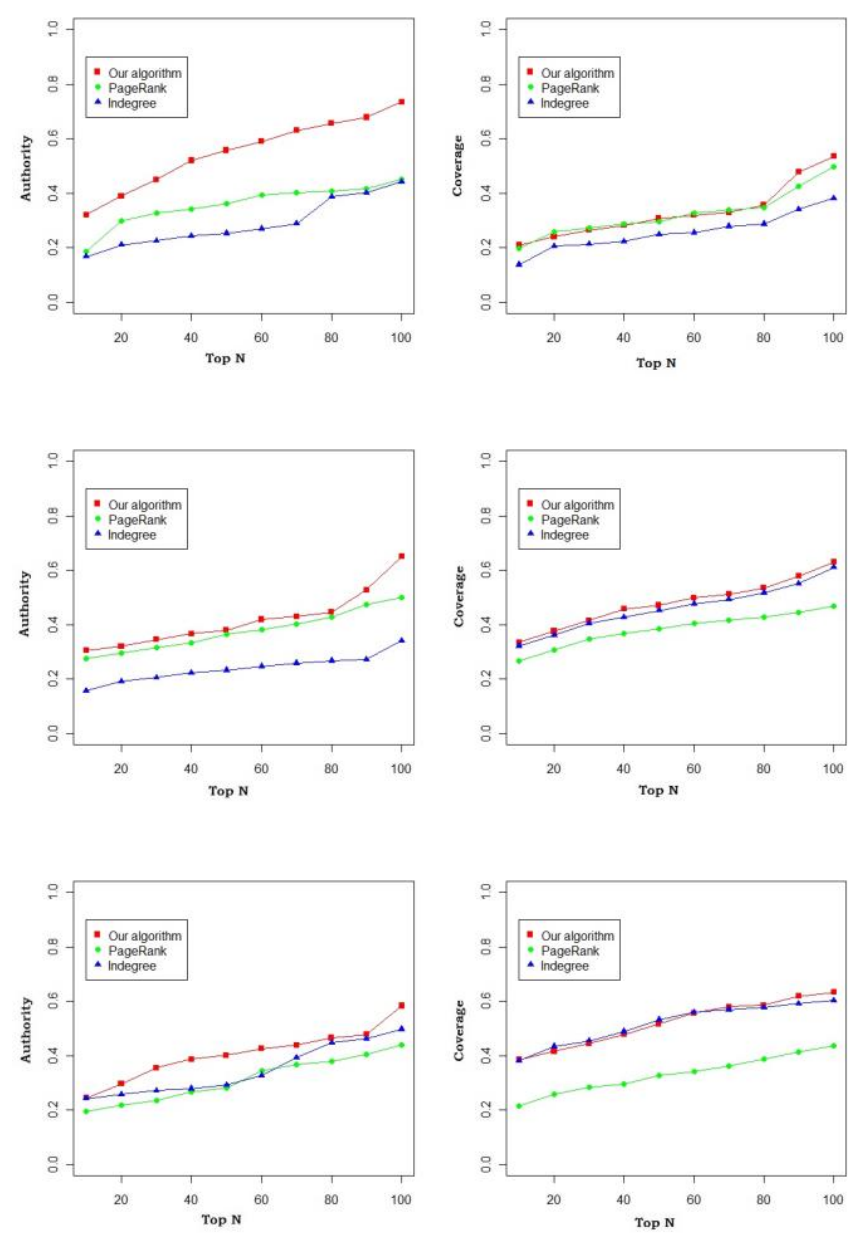

Figure 4. Experiment Analysis on Authority and Coverage in Clothes, Toy and Sports Domain

From Figure 4, we can see that our algorithm significantly outperforms the traditional opinion leaders detecting algorithms on authority and coverage. This is very important for E-commerce companies that can take advantage of opinion leaders to accelerate product diffusion and support increase sales.

\section{Conclusion}

Currently, the E-commerce consumers are at a turning point from transaction-based society to relationship-based society. Word-of-mouth has tremendous impact on Ecommerce decision making. As online social networks have become more popular, consumers often make use of previous consumers' opinions, which is a kind of electronic word-of-mouth. The electronic word-of-mouth from high quality reviews written by previous consumers can have a direct, positive effect on potential consumers' decision making, and this effect can propagate rapidly through online social network by opinion leaders. In this paper, we proposed an algorithm to detect and identify domain-sensitive opinion leaders. Experiments on real world dataset indicate our algorithm is effective. 


\section{Acknowledgement}

This paper is granted by the national training programs of innovation and entrepreneurship for undergraduates (No. 201411065001).

\section{References}

[1] J. Carroll, "Beyond Recommender Systems: Helping People Help Each Other", Addison-Wesley, (2001).

[2] R. Sinha and K. Swearingen, "Comparing Recommendations Made by Online Systems and Friends", In Proceedings of the DELOS-NSF Workshop on Personalization and Recommender Systems in Digital Libraries, Ireland, (2001).

[3] M. Walsh, "Social networking sites fuel E-commerce traffic", (2006), http://publications.mediapost.com/index.cfm?fuseaction=Articles.san\&s=52207.

[4] D. Leonard, "Expert as Negative Opinion Leaders in the Diffusion of a Technological Innovation", Journal of Consumer Research, (1985), pp. 914-926.

[5] F. Feick and L. Price, "The Market Maven, a Diffuser of Marketplace Information", Journal of Marketing, (1987), pp. 83-97.

[6] I. Hawkins, R. Best and A. Coney, "Consumer behavior: Building marketing strategy", McGraw-Hill, Boston, (2004).

[7] J. Arndt, "Role of product-related conversations in the diffusion of a new product", Journal of Marketing Research, (1967), pp. 291-295.

[8] E. Dichter, "How word-of-mouth advertising works", Harvard Business Review, (1966), pp. 131-144

[9] F. Engel, J. Kegerreis and D. Blackwell, "Word-of-mouth communication by the innovator", Journal of Marketing, (1969), pp. 15-19.

[10] J. Carl, "What's all the buzz about? Everyday communication and the relational basis of word-of-mouth and buzz marketing practices", Management Communication Quarterly, (2006), pp. 601-643.

[11] A. Lindgreen and J. Vanhamme, "Viral marketing: The use of surprise", Advances in electronic marketing, (2006), pp. 122-138.

[12] R. Piirto, "The influentials", American Demographics, (1992), pp. 30-38.

[13] C. Walker, "Word of mouth", American Demographics, (1995), pp. 38-45.

[14] W. Gruen, T. Osmonbekov and J. Czaplewski, "eWOM: The impact of customer-to-customer online know-how exchange on customer value and loyalty", Journal of Business Research, (2005), pp. 449-456.

[15] P. Domingos and M. Richardson, "Mining the Network Value of Customers", In Proceedings of the Seventh ACM SIGKDD International Conference on Knowledge Discovery and Data Mining, San Francisco, (2001).

[16] S. Hill, F. Provost and C. Volinsky, "Network-Based Marketing: Identifying Likely Adopters via Consumer Networks", Statistical Science, (2006), pp. 256-276.

[17] P. Sarkar and A. Moore, "Dynamic Social Network Analysis Using Latent Space Models", ACM SIGKDD Explorations Newsletter, (2005), pp. 31-40.

[18] F. Engel, D. Blackwell and W. Miniard, Consumer Behavior, Dryden Press, Orlando, (1990).

[19] X. Song, Y. Chi, K. Hino and B. L. Tseng, "Identifying opinion leaders in the blogosphere", In Proceedings of the Conference on Information and Knowledge Management, Lisboa, (2007).

[20] H. Zhou, D. Zeng and C. Zhang, "Finding leaders from opinion networks", In Proceedings of the Conference on Intelligence and Security Informatics, Dallas, (2009).

[21] J. Weng, E. P. Lim, J. Jiang and Q. He, "TwitterRank: Finding topic-sensitive influential twitterers", In Proceedings of the third ACM International Conference on Web Search and Data Mining, New York, (2010).

[22] P. Jurczyk and E. Agichtein, "Discovering authorities in question answer communities by using link analysis", In Proceedings of the Conference on Information and Knowledge Management, Lisboa, (2007).

\section{Authors}




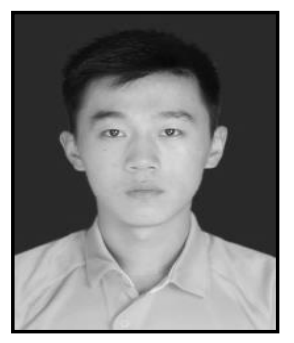

PengPeng Zhang, is currently an undergraduate in the International College at Qingdao University. His main research interests include Electronic Commerce, complex networks, web information retrieval and data mining.

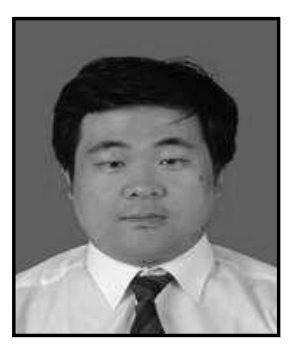

Gengxin Sun, received his Ph.D. degree in Computer Science from Qingdao University, China in 2013. He is currently an Associate Professor in the School of Computer Science and Engineering at Qingdao University. His main research interests include embedded system, operating system, complex networks, web information retrieval and data mining.

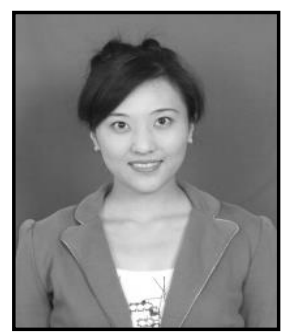

Sheng Bin, received her Ph.D. degree in Computer Science from Shandong University of Science and Technology, China in 2009. She is currently a lecturer in the School of Software Technology at Qingdao University, China. Her main research interests include embedded system, operating system, complex networks, cloud computing and data mining. 
International Journal of $u-$ and e- Service, Science and Technology Vol.8, No. 8 (2015) 